\title{
Dynamic Bayesian Networks for Learning Interactions Between Assistive Robotic Walker and Human Users
}

\author{
Mitesh Patel, Jaime Valls Miro, and Gamini Dissanayake * \\ ARC Centre of Excellence for Autonomous System, University of Technology Sydney \\ 15 Broadway, Ultimo, Sydney, NSW-2007, Australia \\ \{miteshkumar.n.patel, jaime.vallsmiro, gdissa\}@eng.uts.edu.au \\ http://www.cas.edu.au
}

\begin{abstract}
Detection of individuals intentions and actions from a stream of human behaviour is an open problem. Yet for robotic agents to be truly perceived as human-friendly entities they need to respond naturally to the physical interactions with the surrounding environment, most notably with the user. This paper proposes a generative probabilistic approach in the form of Dynamic Bayesian Networks (DBN) to seamlessly account for users attitudes. A model is presented which can learn to recognize a subset of possible actions by the user of a gait stability support power rollator walker, such as standing up, sitting down or assistive strolling, and adapt the behaviour of the device accordingly. The communication between the user and the device is implicit, without any explicit intention such as a keypad or voice. The end result is a decision making mechanism that best matches the users cognitive attitude towards a set of assistive tasks, effectively incorporating the evolving activity model of the user in the process. The proposed framework is evaluated in real-life condition.
\end{abstract}

Key words: Activity Recognition, AI for Robotics, Mobile Robotics, Perception for Human-Robot Interaction, Machine Learning for decision making

\section{INTRODUCTION}

In order to successfully design a robotic system capable of co-existing with humans it is essential to investigate and understand how the human-robot relation and their interactions can be established [5]. In this ever growing field of human-robot interaction (HRI) the use of modern artificial intelligence(AI) tools is becoming more relevant as they are capable of performing complex tasks that require behaviours normally associated with human intelligence. One of the most

* M. Patel, J. V. Miro and G. Dissanayake are with the Faculty of Engineering and IT, University of Technology Sydney (UTS), NSW 2007, Australia 
relevant goals of an "intelligent" user interface for an HRI system is to successfully execute the explicit user commands while at the same time account for the implicit cues that are not so easily observed [10].

Intention recognition, as defined by Heinze's in his doctoral thesis [2], is the process of becoming aware of the intention of another agent (the user in our case) or as the problem of inferring an agent's intention through its actions and their effects in the environment. Due to noisy and partial observations the communication of intentions between the user and agent becomes an important issue. Intention recognition has found its application in many research areas such as user assistance in aviation monitoring [2],human robot co-operation [10] and many other applications.

\section{MOTIVATION}

Assistive technology is increasingly used to offset the impact of impairments resulting from injury, disease, the aging process and related disorders. Typically these technologies have focused on assisting users with mobility impairments. Increasing growth in the number of people with motor disabilities has resulted in considerable research being conducted into developing robotic assistive technologies to address the difficulties that this population faces, and several intelligent systems that use AI and ubiquitous computing techniques have been developed with the older adult population in mind. These include for instance the Nursebot project [9], the PAMM project [1],or the UTS assistive wheelchair project [11]. Researchers in all these projects have explored different avenues to develop robotic assistive technologies using different Probabilistic/Stochastic models.

\section{PREVIOUS WORK}

Probabilistic Networks (PN), also known as Belief Networks, has become a popular tool in the AI community as they can represent a complex system using graphical models [4]. Due to the amount of uncertainty involved in designing user assistive systems, researchers have explored the possibility of using various stochastic models like Markov Decision Process Models(MDP)/Partially Observable Markov Decision Process Models (POMDPs) [6], [11],Hidden Markov Models (HMM),Bayesian Network (BN) [3] and Dynamic Bayesian Network (DBN) [12]. The typical temporal dynamics of an assistive system consists of a sensorial system and an engine which, after learning, infers the user behaviour from the newly acquired data.

BNs, HMMs, hybrid HMMs (H-HMMs), auto-regressive HMMs (A-HMM), factorial-HMMs (F-HMMs) can be viewed as variants of DBN [7] as all these models can be represented using graphical models. BN being a static network only works with results from a single slice of time and hence does not work for analyzing an evolving system that changes its modelling behaviours over time.

POMDPs and DBNs are stochastic models for representing the evolution of variables over time. It consists of sequence of time-slices where each time 
slice contains set of variables representing the state at the current time [12]. In POMDP a control policy computes an action after every observation such that in the long-run the expected utility is maximized [13]. They are advantageous in modelling systems where the expected predictions or results are maximized in the long term. On the other hand, DBNs provides a framework where expected predictions or results are maximized in the immediate time slice and they are computationally more efficient.

\section{PROPOSITION}

In this paper we propose to use DBN as a probabilistic decision making AI model suitable to be employed in mobility rehabilitative tasks, with the emphasis on the interactions between the machine and the human in the loop. The aim of this system is to understand and recognize the intention of a user (among a given set of alternatives) while he/she is using an instrumented powered rollator walker. The intention of a user is generally a hidden state in that it cannot be directly observed. Hence, the robotic agent has the task of deciding what the user really wants to do based on a minimalistic set of unobtrusive indicative inputs from the user.

\section{THE DBN FRAMEWORK}

Dynamic Bayesian Networks are a branch of Bayesian Networks for modelling sequential data.They are represented by directed acyclic graphs (DAG) in which nodes are variables and arcs show the conditional independencies among the variables.Each node on the net has a probability table associated containing the conditional probabilities of each of the values that node can take with respect to each of the possible combination of its parent nodes. A typical DBN is depicted in Figure 1. The network only considers the discrete-time stochastic process, and it increases the index time $t$ by one every time a new observation is recorded [7].

$$
P\left(Z_{t} / Z_{t-1}\right)=\prod_{i=1}^{N} P\left(Z_{t}^{i} \mid P a\left(Z_{t}^{i}\right)\right)
$$

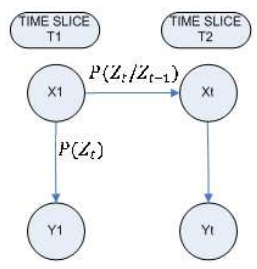

Fig. 1. DBN Structure unrolled for two time slices. 
A DBN is defined to be a pair, $\left(B_{1}, B_{->}\right)$, where $B_{1}$ is a Bayesian Network which defines the prior $\mathrm{P}\left(Z_{1}\right)$, and $B_{->}$is a two slice temporal Bayes net which defines $\mathrm{P}\left(Z_{t} / Z_{t-1}\right)$ by means of a directed acyclic graph as given by Equation 1 . Except for the first nodes in the first time slice, all the other subsequent nodes have an associated conditional probability distribution (CPD).

To construct a DBN we need to define three clusters of information: the prior distributions which can be termed as the initial probabilities of the state variables, the transition model and the conditional probability distribution between states, and the sensor model. To specify the transition probability model we must also specify the topology of the connections between successive slices and between the states and evidence variables in each time slice.

\section{EXPERIMENTAL SETUP}

The power walker employed is displayed in Figure 3(a). It is a modified commercial rollator walking frame with four wheels. The base frame has been instrumented with actuators and incremental encoders to the two rear wheels (front wheels are passive), two infra-red (IRs) proximity sensors to detect the presence of the user, four strain gauges (SGs) to detect indicative pressure, two push button switches on each of the walker's handle-bar to recognize the actual intention of the user, a low-level micro-controller and a high-level control computer.

The installed strain gauges are two Micro Measurements 120UR. The differential force between the vertical axis of each handle-bar are used to establish whether the user is holding on the handle-bar and is in readiness to start some task such as sitting down, standing up or ambulation.

The IR subsystem sensor is made up of two Sharp GP2Y0A02YK, which are used to estimate whether the driving user in standing behind the walker and how far they are from it. The motorized actuation subsystem is based around two powerful Matsushita Electric GMX-8MC045A 24VDC reversible gear-head motor with optical encoder and rotary mechanical couplings, driven using a national semiconductor LMD18200 3A, 55V H-Bridge motor driver.

A compact Hokuyo URG-04LX laser range finder is also incorporated in the design for localization and reactive navigation. The URG-04LX is able to report

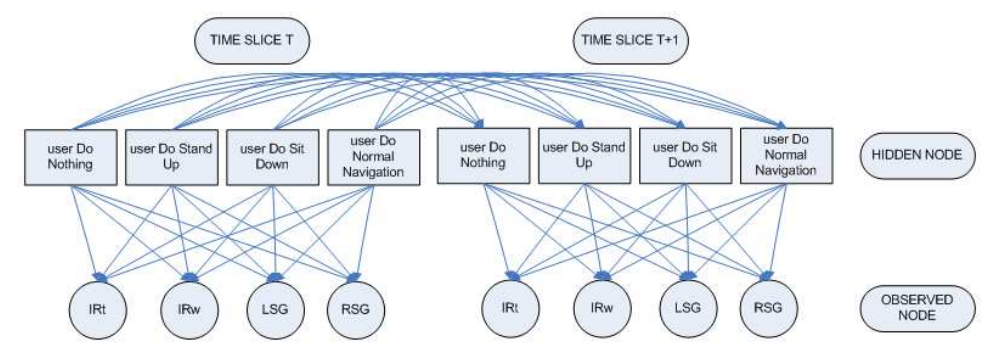

Fig. 2. DBN Model for our application when unrolled for two time slices. 

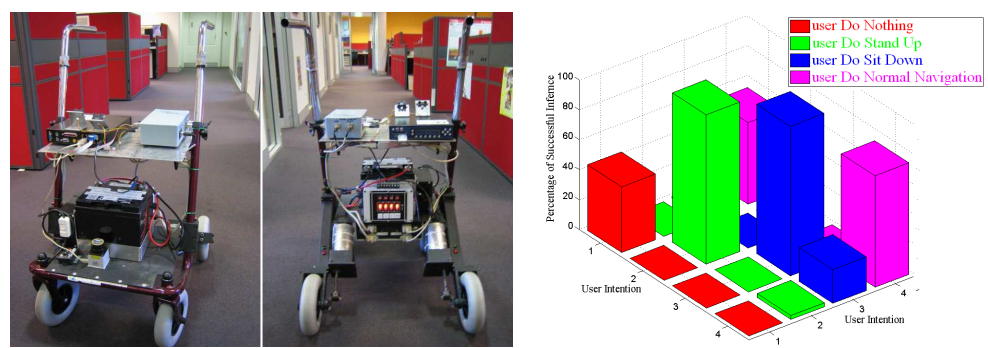

(a) Front and Rear View of the (b) Offline inference results of the Walker

DBN Model

Fig. 3. (a) The instrumented rollator walker platform showing the laser range finder at the front, the infra-red proximity sensors on top of the black PC controller, servomotors and encoders and force sensors mounted on the handlebars and (b) Percentage accuracy of the DBN Model when tested offline in correctly inferring user intention

ranges from $0.02 \mathrm{~m}$ to $4.0 \mathrm{~m}(0.0001 \mathrm{~m}$ resolution $)$ in a $240^{\circ}$ arc. Its power consumption, $500 \mathrm{MA} @ 5 \mathrm{~V}$, makes it a natural choice for battery operated vehicles. The localization data obtained using the Hokuyo laser is used to plot the user intention at a particular location as shown in Figure 4.

The two push button switches installed on handles of the walker are used to record the actual intention of the user. This was done to compare the real-time intention of the user with the inference of the DBN model. The users were asked to press the correct switch while executing their intended action.

\subsection{DBN as a Decision Making Agent on Walker}

The desired DBN model has four observed nodes which represent the data collected from the four sensors on the walker and four hidden nodes which predicts the temporal state of the user. Figure 2 shows the intra- and inter- connections between nodes (in the example presented in this paper), which are then repeated over subsequent time slices.The four temporal states are Do Nothing, Stand Up, Sit Down, Normal Navigation(Power Assisted Strolling). The data from the sensor system being noisy, its corresponding nodes in the DBN model are represented as continuous gaussian nodes. The hidden node which predicts the user intention is discrete. The hidden nodes between time slices are interconnected as the future prediction of the user intention depends on the previous intention.

The transition probabilities between time slices were defined using various techniques viz. based on past experiences [6], common laws of operation and performing simulation. Specifically, we collected data from user trial runs while executing the tasks on the walker system, and also reflected what was perceived as accepted behavioural transitions (e.g., users will not sit down immediately after standing up, probability of the user to sit down immediately after standing up will be low...). 


\section{EXPERIMENTAL RESULTS}

The proposed DBN algorithm was evaluated within the domain of our office environment, a typical working space with desks, cubicles, people walking about, open meeting areas, corridors,etc. A 2D bird's eye view of the map can be seen in Figure 4 overlaid with some of the results detailed. The initial testing of the DBN model was done in a simulation environment in Matlab. Simulations in matlab environment also gave us an opportunity to derive the most optimal transition probability matrix.

Off-line testing of the DBN model was done after recording the real-time data from the sensor system while asking the user to perform different tasks. The DBN was designed to recognize one of the four user intention. Data was recorded from five different users with distinctive physical characteristics and behaviours. Each user was asked to manoeuver the walker such that they perform all the intended task during the course so that sensor data for all the user intentions/actions could be recorded. Data sets recorded for each user were merged manually. Each user data set consists of 65 samples for each action, from which 50 samples were used for training the DBN model and 15 (not used in training) for testing. The total number for all users was 250 samples for each action used for training, and correspondingly 75 samples used for testing. The Matlab BNT toolbox [7] was used to design the DBN and perform offline testing. Figures 3(b) graphically depict the accuracy of the inference engine, which indicates successful recognition rates in the order of $81 \%$.It can be seen in Figure 3(b) that the DBN model can correctly predict more distinctive behaviours, such as when performing the action of sitting down and standing up, while at times it gets confused between the Do Nothing and Normal Navigation given the more subtle driving indication variability from user to user as sensed primarily by the strain gauges.

The promising results of the offline testing, encouraged us to test the DBN model in real-time. We used $\mathrm{C}++$ programming environment and Mocapy++ toolbox [8] to perform real-time testing. The data set, used for offline simulation was used to train the DBN model along with the most optimal transition matrix obtained during simulations. The trained DBN model was used to infer human intention based on the realtime data available from the sensors. Results in Figures 4 shows the comparison of the actual user intention (recorded by pressing the correct combination of the push button switches) and that inferred by the DBN model while the walker is being manoeuvred around the office by the user. A total of 496 samples of observation were collected from the sensors while the

\begin{tabular}{|c|c|c|c|}
\hline Users & Total Samples & false Inference By DBN & Accuracy \\
\hline \hline User 1 & 179 & 29 & $83.79 \%$ \\
User 2 & 317 & 12 & $96.21 \%$ \\
\hline
\end{tabular}

Table 1. Inference results obtained from two different users while manoeuvering the walker in a office environment 


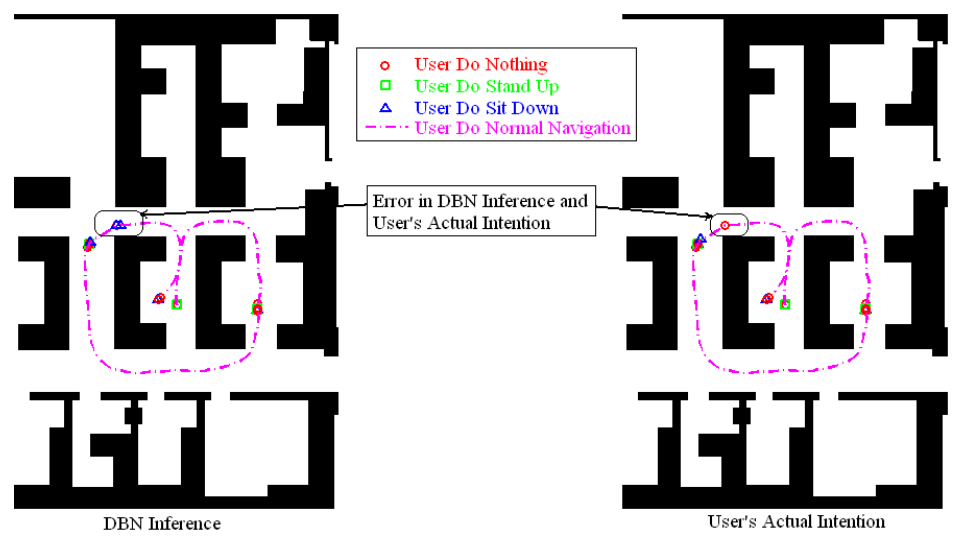

Fig. 4. 2D view of DBN Inference and Users' Actual Intention. The various tasks are depicted in different colours

user was performing the task shown in Figure 4. The average recognition rates achieved when using the system for two users (one user being new to the system and another being known to the system) was in the order of $91.73 \%$. A detailed break down of the results with each individual user is shown in table 1 . The accuracy is higher with User 2 as he is the designer of the DBN model and the overall walker system, hence he has a better understanding of the working of the system. The accuracy of the DBN model does not vary as much when tested with a user, new to the system.

\section{CONCLUSION AND FUTURE WORK}

In this paper a generic DBN framework capable of unobtrusively recognize a set of possible actions by the users of a gait stability support power rollator walker has been presented. Since user intentions don't happen in a fixed sequence hence a stochastic approach has been used to model the relation of intentions and corresponding sensor measurements. The successful implementation of the proposed stochastic framework have shown an intentions recognition mechanism that can be readily adapted to allow for more natural human robot interactions. The DBN model being highly flexible, it can be easily extended by adding new intentions and observations to the model. In future we plan to fuse the DBN model along with other static classifiers ("SVM", "BN") such that the classifier can be used to provide the low level prediction based on the data available from the sensors, whereas the DBN engine can be used for high level user state inference. 


\section{ACKNOWLEDGMENT}

This work is supported by the Australian Research Council (ARC) through its Centre of Excellence programme, and by the New South Wales State Government. The ARC Centre of Excellence for Autonomous Systems (CAS) is a partnership between the University of Technology Sydney, the University of Sydney and the University of New South Wales.

\section{References}

1. Dubowsky, S., Génot, F., Godding, S., Kozono, H., Skwersky, A., Yu, H., Yu, L.S.: PAMM - A robotic aid to the elderly for mobility assistance and monitoring: A helping-hand for the elderly. In: IEEE International Conference on Robotics and Automation. pp. 570-576. IEEE (2000)

2. Heinze, C.: Modeling Intention Recognition for Intelligent Agent Systems. Ph.D. thesis, The University of Melbourne (2003)

3. Hong, J.H., Song, Y.S., Cho, S.B.: A hierarchical bayesian network for mixedinitiative human-robot interaction. In: Proceedings of the 2005 IEEE International Conference on Robotics and Automation,(ICRA 2005). pp. 3808-3813 (April 2005)

4. Jensen, F.V.: An Introduciton to Bayesian Networks. UCL Press (1996)

5. Lund, H.: Modern artificial intelligence for human-robot interaction. Journal of Proceedings of the IEEE 92(11), 1821-1838 (Nov 2004)

6. Miro, J.V., Osswald, V., Patel, M., Dissanayake, G.: Robotic assistance with attitude: A mobility agent for motor function rehabilitation and ambulation support. In: IEEE International Conference on Rehabilitation Robotics, ICORR 2009. pp. 529-534 (June 2009)

7. Murphy, K.P.: Dynamic Bayesian Networks: Representation, Inference and Learning. Ph.D. thesis, University of Califronia, Berkeley (2002)

8. Paluszewski, M., Hamelryck, T.: Mocapy++ - a toolkit for inference and learning in dynamic bayesian networks. BMC Bioinformatics 11(1), 126 (2010)

9. Pineau, J., Montemerlo, M., Pollack, M.E., Roy, N., Thrun, S.: Towards robotic assistants in nursing homes: Challenges and results. Robotics and Autonomous Systems 42(3-4), 271-281 (2003), http://dx.doi.org/10.1016/S0921-8890(02) 00381-0

10. Schrempf, O.C., Hanebeck, U.D.: A generic model for estimating user intentions in human-robot cooperation. In: Proceedings of the 2nd International Conference on Informatics in Control, Automation and Robotics (ICINCO 2005). vol. 3, pp. 251-256. Barcelona, Spain (sep 2005)

11. Taha, T., Miro, J.V., Dissanayake, G.: Pomdp-based long-term user intention prediction for wheelchair navigation. In: IEEE International Conference on Robotics and Automation,(ICRA 2008). pp. 3920-3925 (May 2008)

12. Tahboub, K.A.: Intelligent human-machine interaction based on dynamic bayesian networks probabilistic intention recognition. Journal of Intelligent and Robotic Systems (2005) 45(1), 31-52 (2004)

13. Theocharous, G., Mannor, S., Shah, N., Gandhi, P., Kveton, B., Siddiqi, S., Yu, C.H.: Machine learning for adaptive power management. Intel Technology Journal 10(4) (2006) 